\title{
A SYNTHESIS OF 6-DEOXY-6-FLUOROSUCROSE SUITABLE FOR PET
}

\author{
APPLICATIONS \\ Xuefeng Gao, Vikram Gaddam and Michael Harmata*
}

\begin{abstract}
A new route to 6-deoxy-6-fluorosucrose has been developed. The process proceeds in 8 linear steps in $25 \%$ overall yield from sucrose. The steps incorporating fluorine and subsequent deprotection are quite rapid, making the procedure useful in the context of ${ }^{18} \mathrm{~F}$ labelling for PET applications.
\end{abstract}

Keywords: fluorination, fluorosucrose, fluorosugar, Kryptofix [2.2.2]

\section{Introduction}

In the context of our interest in examining sucrose transport in plants, we recently reported syntheses of both 6'-deoxy-6'-fluorosucrose ${ }^{1}$ and 1'-deoxy-1'-fluorosucrose. ${ }^{2}$ These compounds are currently being used in their "hot" $\left({ }^{18} \mathrm{~F}\right)$ form in imaging studies concerning sucrose transport in maize. ${ }^{3}$ In order to get more complete information on the nature of sucrose transport, we require a sucrose derivative that is functionalized in the glucose half of the molecule. For this purpose, we chose 6-deoxy-6-fluorosucrose (6-F-sucrose).

A synthesis of 6-F-sucrose was reported by Eklund and Robyt a number of years ago. ${ }^{4}$ The key step of the process involved treatment of the sucrose derivative $\mathbf{1}$ with diethylaminosulfur trifluoride (DAST) in dichloromethane at $30{ }^{\circ} \mathrm{C}$ for $22 \mathrm{~h}$. While the overall yield from sucrose 
was $11 \%$, the length of time needed for fluorination was much too long for our needs. We thus set out to develop an improved synthesis.

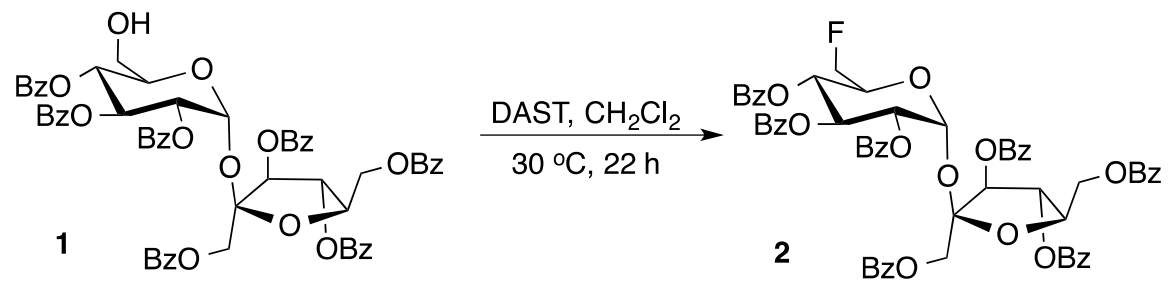

Scheme 1. Fluorination step in the Eklund-Robyt approach to 6-F-sucrose.

\section{Results and Discussion}
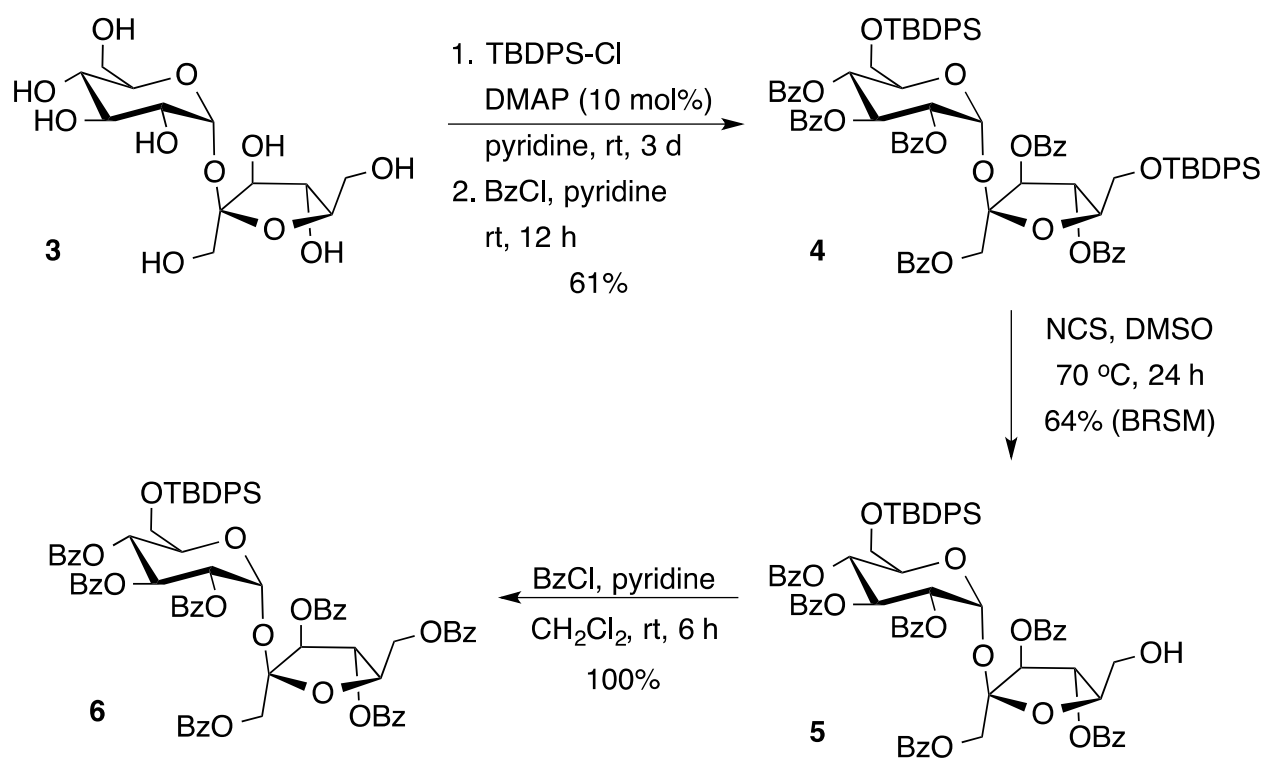
We have developed a synthetic protocol to 6-deoxy-6-fluorosucrose that is quite suitable for application to an ${ }^{18} \mathrm{~F}$-labeled species. We will apply the hot compound to studies of sucrose transport in maize. Results will be reported in due course.

\section{Experimental section}

\subsection{General methods}

All reactions were carried out in oven-dried or flame-dried flasks under an atmosphere of argon. Acetonitrile, dichloromethane and methanol were freshly distilled over calcium hydride. Analytical thin layer chromatography was performed on normal and reverse phase silica gel plates impregnated with a UV indicator. Flash chromatography was carried out using 230-400 mesh silica gel with HPLC grade solvents. ${ }^{1} \mathrm{H}$ and ${ }^{13} \mathrm{C}$ NMR spectra were recorded on a Bruker DRX 500 (500 MHz for ${ }^{1} \mathrm{H}, 125 \mathrm{MHz}$ for ${ }^{13} \mathrm{C}$ ) spectrometer in $\mathrm{CDCl}_{3}$ (TMS as internal standard) or $\mathrm{D}_{2} \mathrm{O} .{ }^{19} \mathrm{~F}$ NMR was recorded on a Bruker ARX-250 (235.3 $\mathrm{MHz}$ for $\left.{ }^{19} \mathrm{~F}\right)$ spectrometer in $\mathrm{D}_{2} \mathrm{O}$ $\left(\mathrm{CFCl}_{3}\right.$ as external standard). Melting points were determined with a Fisher-Johns melting point apparatus. Infrared spectra were recorded on a Perkin Elmer 1600 series FT-IR spectrometer. High-resolution mass spectra were performed by College of Science Major Instrumentation Center, Old Dominion University, with a Bruker 12 Tesla APEX-Qe FTICR-MS. Optical rotations were measured with a JASCD DIP-370 digital polarimeter.

\subsection{6,6'-(bis)-O-tert-butyldiphenylsilylsucrose}

To a solution of $20 \mathrm{~g}$ (58.4 mmol) of sucrose in $200 \mathrm{~mL}$ pyridine was added $200 \mathrm{mg}$ of DMAP (10 mol \%), followed by $36.3 \mathrm{~mL}$ (140.2 mmol, 2.2 equiv) of TBDPSCl. The solution was stirred at $\mathrm{rt}$ for 3 days, then concentrated under reduced pressure. One hundred $\mathrm{mL}$ of EtOAc was added, and the mixture was concentrated under reduced pressure. The residue was purified by 
column chromatography using 1-2.5\% $\mathrm{MeOH}$ in EtOAc, which resulted in $29 \mathrm{~g}$ (35.6 mmol, $61 \%$ yield) of product as a white solid. This was further purified by recrystallization from EtOAc, affording $14.85 \mathrm{~g}$ (18.1 mmol, 31\% yield) of pure product as a white solid, mp 208-209 ${ }^{\circ} \mathrm{C} .{ }^{1} \mathrm{H}$ NMR $\left(500 \mathrm{MHz}, \mathrm{CDCl}_{3}\right) \delta$ 7.67-7.56 (m, 8H), 7.40-7.20 (m, 12H), 5.41 (d, $J_{1,2}=3.5 \mathrm{~Hz}$, 1H, H-1), 4.09 (t, $\left.J_{3^{\prime}, 4^{\prime}}=7.5 \mathrm{~Hz}, 1 \mathrm{H}, \mathrm{H}^{\prime} 3^{\prime}\right), 4.02$ (t, $\left.J_{3^{\prime} 4^{\prime}}=J_{4^{\prime}, 5^{\prime}}=7.5 \mathrm{~Hz}, 1 \mathrm{H}, \mathrm{H}-4^{\prime}\right), 3.91-3.73$

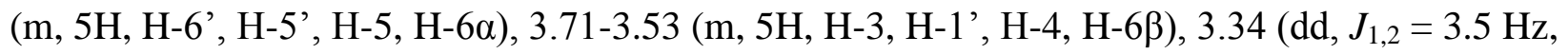
$\left.J_{2,3}=9.5 \mathrm{~Hz}, 1 \mathrm{H}, \mathrm{H}-2\right), 0.96(\mathrm{~s}, 9 \mathrm{H}), 0.95(\mathrm{~s}, 9 \mathrm{H}) ;{ }^{13} \mathrm{C} \mathrm{NMR}\left(125 \mathrm{MHz}, \mathrm{CD}_{3} \mathrm{OD}\right) \delta 136.80$, $136.77,136.74,136.71,134.83,134.51,134.49,134.48,130.78,130.76,130.71,130.69,128.81$, 128.78, 128.73, 128.68, 105.95 (C-2'), 93.42 (C-1), 83.95 (C-5'), 79.49 (C-3'), 77.06 (C-4'), 74.95 (C-3), 74.20 (C-5), 73.33 (C-2), 71.33 (C-4), 66.46 (C-6’), 64.44 (C-6), 64.36 (C-1'), 27.34, 20.09, 20.00; IR $\left(\mathrm{cm}^{-1}\right) 3395,3305,3187,3070,2976,2928,2884,2855,1588,1472$, 1461, 1426, 1389, 1363, 1339, 1322, 1300, 1266, 1188,1152, 1102, 1046, 1013, 989, 960, 937, 910, 880, 824, 800, 741, 701, 689; HRMS m/z calcd for $\left(\mathrm{C}_{44} \mathrm{H}_{58} \mathrm{O}_{11} \mathrm{Si}_{2}\right) \mathrm{Na}^{+}$841.3410, found $841.3404 ;[\alpha]_{D}^{20}=25.0(\mathrm{c} 1.00, \mathrm{MeOH})$.

\section{3. $6,6^{\prime}$-(bis)-O-tert-butyldiphenylsilyl-2,3,4,1',3',4'-hexa- $O$-benzoylsucrose (4)}

To a solution of $14.85 \mathrm{~g}$ (18.1 mmol) of 6,6'-(bis)-O-tert-butyldiphenylsilylsucrose in $90.7 \mathrm{~mL}$ of $\mathrm{CH}_{2} \mathrm{Cl}_{2}$ was added $17.6 \mathrm{~mL}$ of pyridine $(218 \mathrm{mmol}, 12$ equiv). The solution was stirred vigorously at $\mathrm{rt}$ for $10 \mathrm{~min}$, then $25.3 \mathrm{~mL}$ (218 mmol 12 equiv) of benzoyl chloride was added and the mixture was stirred at $\mathrm{rt}$ for $12 \mathrm{~h}$. The solution was then washed with water and brine, dried with $\mathrm{MgSO}_{4}$ and concentrated under reduced pressure. One hundred $\mathrm{mL}$ of hexanes were added, and the mixture was concentrated under reduced pressure. The residue was purified by column chromatography (5-20\% EtOAc in hexanes), affording $26.1 \mathrm{~g}$ (18.1 mmol, 100\% yield) of a white solid, mp $159-160{ }^{\circ} \mathrm{C}$ (recrystallized from $\left.\mathrm{MeOH}\right) .{ }^{1} \mathrm{H} \mathrm{NMR}\left(500 \mathrm{MHz}, \mathrm{CDCl}_{3}\right) \delta$ 
$8.03(\mathrm{~d}, J=7.5 \mathrm{~Hz}, 2 \mathrm{H}), 7.96(\mathrm{~d}, J=8.5 \mathrm{~Hz}, 2 \mathrm{H}), 7.94(\mathrm{~d}, J=9 \mathrm{~Hz}, 2 \mathrm{H}), 7.88(\mathrm{~d}, J=7.5 \mathrm{~Hz}$ 2H), $7.81(\mathrm{~d}, J=8.0 \mathrm{~Hz}, 2 \mathrm{H}), 7.79(\mathrm{~d}, J=8.5 \mathrm{~Hz}, 2 \mathrm{H}), 7.66(\mathrm{~d}, J=7.0 \mathrm{~Hz}, 2 \mathrm{H}), 7.60-7.12(\mathrm{~m}$, $34 \mathrm{H}), 7.06(\mathrm{t}, J=7.7 \mathrm{~Hz}, 2 \mathrm{H}), 6.10\left(\mathrm{t}, J_{2,3}=J_{3,4}=10.0 \mathrm{~Hz}, 1 \mathrm{H}, \mathrm{H}-3\right), 6.02\left(\mathrm{~d}, J_{1,2}=3.0 \mathrm{~Hz}, 1 \mathrm{H}\right.$, $\mathrm{H}-1), 5.96\left(\mathrm{t}, J_{3,4}=J_{4,5}=10.0 \mathrm{~Hz}, 1 \mathrm{H}, \mathrm{H}-4\right), 5.95\left(\mathrm{t}, J_{3^{\prime}, 4^{\prime}}=J_{4^{\prime}, 5^{\prime}}=6.5 \mathrm{~Hz}, 1 \mathrm{H}, \mathrm{H}-4^{\prime}\right), 5.86(\mathrm{~d}$, $\left.J_{3^{\prime}, 4^{\prime}}=6.0 \mathrm{~Hz}, 1 \mathrm{H}, \mathrm{H}-3^{\prime}\right), 5.40\left(\mathrm{dd}, J_{1,2}=3.5 \mathrm{~Hz}, J_{2,3}=10.5 \mathrm{~Hz}, 1 \mathrm{H}, \mathrm{H}-2\right), 4.63\left(\mathrm{~d}, J_{1^{\prime} \alpha, 1^{\prime} \beta}=12.0\right.$ $\left.\mathrm{Hz}, 1 \mathrm{H}, \mathrm{H}-1^{\prime} \alpha\right), 4.58\left(\mathrm{~d}, J_{1^{\prime} \alpha, 1^{\prime} \beta}=12.0 \mathrm{~Hz}, 1 \mathrm{H}, \mathrm{H}-1^{\prime} \beta\right), 4.31\left(\mathrm{~m}, 2 \mathrm{H}, \mathrm{H}-5, \mathrm{H}-5^{\prime}\right), 3.92\left(\mathrm{dd}, J_{5^{\prime}, 6^{\prime} \alpha}=\right.$ $\left.5.5 \mathrm{~Hz}, J_{6^{\prime}} \alpha, 6^{\prime} \beta=11.0 \mathrm{~Hz}, 1 \mathrm{H}, \mathrm{H}-6^{\prime} \alpha\right), 3.88\left(\mathrm{dd}, J_{5^{\prime}, 6^{\prime} \beta}=5.5, J_{6^{\prime} \alpha, 6^{\prime} \beta}=11.0 \mathrm{~Hz}, 1 \mathrm{H}, \mathrm{H}-6^{\prime} \beta\right), 3.52$ $\left(\mathrm{d}, J_{6 \alpha, 6 \beta}=12.0 \mathrm{~Hz}, 1 \mathrm{H}, \mathrm{H}-6 \alpha\right), 3.43\left(\mathrm{~d}, J_{6 \alpha, 6 \beta}=12.0 \mathrm{~Hz}, 1 \mathrm{H}, \mathrm{H}-6 \beta\right), .1 .01(\mathrm{~s}, 9 \mathrm{H}), 0.97(\mathrm{~s}, 9 \mathrm{H})$; ${ }^{13} \mathrm{C}$ NMR $\left(125 \mathrm{MHz}, \mathrm{CDCl}_{3}\right) \delta 165.72,165.65,165.55,165.44,164.96,164.69,135.71,135.54$, 135.50, 135.47, 133.34, 133.21, 133.08, 133.02, 132.96, 132.93, 132.81, 132.77, 132.70, 130.07, $129.88,129.81,129.78,129.72,129.65,129.55,129.49,129.43,129.36,129.18,129.04,128.87$, 128.52, 128.37, 128.27, 128.22, 128.17, 127.67, 127.64, 127.60, 127.41, 103.93 (C-2'), 90.71 (C-1), 81.17 (C-5'), 77.75 (C-3'), 75.26 (C-4'), 71.40 (C-2, C-5), 70.96 (C-3), 68.10 (C-4), 65.31 (C-1'), 63.74 (C-6'), 61.00 (C-6), 26.68, 26.64, 19.11, 19.02; IR ( $\left.\mathrm{cm}^{-1}\right)$ 3071, 2959, 2931, 2858, 1966, 1733, 1602, 1585, 1492, 1472, 1451, 1428, 1390, 1362, 1315, 1265, 1177, 1106, 1070, 1026, 824, 802, 739, 708; HRMS m/z calcd for $\left(\mathrm{C}_{86} \mathrm{H}_{82} \mathrm{O}_{17} \mathrm{Si}_{2}\right) \mathrm{Na}^{+} 1465.4983$, found 1465.4968; $[\alpha]_{D}^{20}=8.2\left(\mathrm{c} 1.00, \mathrm{CHCl}_{3}\right)$.

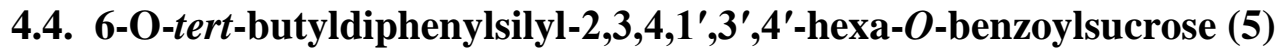

To a solution of $26.1 \mathrm{~g}$ of $4(18.1 \mathrm{mmol})$ in $181 \mathrm{~mL}$ of DMSO $(0.1 \mathrm{M}), 7.25 \mathrm{~g}(54.3 \mathrm{mmol}, 3$ equiv) of $\mathrm{N}$-chlorosuccinimide (NCS) was added. The reaction was stirred at $70{ }^{\circ} \mathrm{C}$ for $24 \mathrm{~h}$ and monitored by TLC (small amount of solution was transferred to a test tube, diluted with EtOAc and washed with water; analyzed by TLC with 30\% EtOAc in hexanes, for $4 R_{f}=0.5$, for $5 R_{f}=$ 0.43.) To the reaction was added $200 \mathrm{~mL}$ of EtOAc at $\mathrm{rt}$ and the mixture was stirred for $1 \mathrm{~min}$. 
The EtOAc layer was washed with water, brine, and dried over $\mathrm{MgSO}_{4}$. The crude product was purified by flash chromatography $\left(\mathrm{SiO}_{2}, 30 \%\right.$ EtOAc/hexanes) affording $12 \mathrm{~g}$ of a white solid (55\%, 64\% yield based on recovered starting material), along with $3.46 \mathrm{~g}$ recovered starting material. ${ }^{1} \mathrm{H}$ NMR $\left(500 \mathrm{MHz}, \mathrm{CDCl}_{3}\right) \delta 8.12(\mathrm{~d}, J=7.5 \mathrm{~Hz}, 2 \mathrm{H}), 8.00(\mathrm{~d}, J=8.0 \mathrm{~Hz}, 2 \mathrm{H}), 7.98$ $(\mathrm{d}, J=8.0 \mathrm{~Hz}, 2 \mathrm{H}), 7.92(\mathrm{~d}, J=7.5 \mathrm{~Hz}, 2 \mathrm{H}), 7.89(\mathrm{~d}, J=7.5 \mathrm{~Hz}, 2 \mathrm{H}), 7.88(\mathrm{~d}, J=7.0 \mathrm{~Hz}, 2 \mathrm{H})$, $7.77(\mathrm{~d}, J=3.5 \mathrm{~Hz}, 2 \mathrm{H}), 7.56(\mathrm{~d}, J=7.5 \mathrm{~Hz}, 2 \mathrm{H}), 7.54-7.32(\mathrm{~m}, 13 \mathrm{H}), 7.32-7.22(\mathrm{~m}, 7 \mathrm{H}), 7.15$ $(\mathrm{d}, J=7.5 \mathrm{~Hz}, 2 \mathrm{H}), 7.14(\mathrm{~d}, J=6.5 \mathrm{~Hz}, 2 \mathrm{H}), 6.26-6.20(\mathrm{~m}, 2 \mathrm{H}, \mathrm{H}-1, \mathrm{H}-3), 6.08\left(\mathrm{t}, J_{3,4}=J_{4,5}=\right.$ $10.0 \mathrm{~Hz}, 1 \mathrm{H}, \mathrm{H}-4), 6.05-5.99$ (m, 2H, H-3', H-4'), 5.41 (dd, $J_{2,1}=3.0 \mathrm{~Hz}, J_{2,3}=10.5 \mathrm{~Hz}, 1 \mathrm{H}, \mathrm{H}-$ 2), 4.59 (s, 2H, H-1'), 4.38 (d, J J 5 = 10.0 Hz, 1H, H-5), 4.25 (s, 1H, H-5'), 3.86-3.80 (m, 1H, H$\left.6^{\prime} \alpha\right), 3.80-3.72(\mathrm{~m}, 1 \mathrm{H}, \mathrm{H}-6$ ' $\beta), 3.66$ (s, 2H, H-6), 3.05 (t, $\left.J_{6^{\prime}, \mathrm{OH}}=7.5 \mathrm{~Hz}, 1 \mathrm{H}, \mathrm{H}-\mathrm{OH}\right), 1.09$ (s, 9H). ${ }^{13} \mathrm{C}$ NMR $\left(125 \mathrm{MHz}, \mathrm{CDCl}_{3}\right) \delta 166.53,165.62,165.47,165.43,165.18,164.59,135.74$, $135.47,133.46,133.34,133.02,133.00,132.84,132.76,132.73,129.99,129.71,129.67,129.58$ $129.42,129.28,129.27,129.19,128.81,128.72,128.49,128.43,128.40,128.28,128.19,128.13$ 127.58, 127.41, 103.77 (C-2'), 90.70 (C-1), 81.71 (C-5'), 77.80 (C-3’), 74.41 (C-4'), 71.97 (C2), 71.84 (C-5), 70.33 (C-3), 67.75 (C-4), 65.60 (C-1'), 61.03 (C-6), 60.83 (C-6’), 26.64, 19.09; IR $\left(\mathrm{cm}^{-1}\right)$ 3512, 3071, 2931, 2858, 1733, 1602, 1585, 1492, 1472, 1451, 1428, 1390, 1315, 1267, $1178,1160,1095,1070,1026,850,824,802,739,708,686$; HRMS m/z calcd for $\left(\mathrm{C}_{70} \mathrm{H}_{64} \mathrm{O}_{17} \mathrm{Si}\right) \mathrm{Na}^{+}$1227.3805, found 1227.3797; $[\alpha]_{D}^{20}=21.8\left(\mathrm{c} 1.00, \mathrm{CHCl}_{3}\right)$.

\subsection{6-O-tert-butyldiphenylsilyl-2,3,4,1',3',4',6'-hepta-O-benzoylsucrose (6)}

To a solution of $12.36 \mathrm{~g}(10.25 \mathrm{mmol})$ of 5 in $51.3 \mathrm{~mL} \mathrm{CH}_{2} \mathrm{Cl}_{2}(0.2 \mathrm{M})$ was added $2.49 \mathrm{~mL}$ of pyridine (30.76 mmol, 3.0 equiv) followed by $3.57 \mathrm{~mL}$ of benzoyl chloride ( $30.76 \mathrm{mmol}, 3.0$ equiv) at room temperature. The reaction was stirred at $\mathrm{rt}$ for $6 \mathrm{~h}$, then the solvent was removed under reduced pressure. The residue was purified by flash chromatography $\left(\mathrm{SiO}_{2}, 20 \%\right.$ 
EtOAc/hexane), which resulted $13.4 \mathrm{~g}(10.25 \mathrm{mmol})$ of $\mathbf{6}$, solid foam $\mathrm{mp} 87-90{ }^{\circ} \mathrm{C} ; \mathrm{R}_{\mathrm{f}}=0.48$, 30\% EtOAc/hexanes; ${ }^{1} \mathrm{H}$ NMR (500 MHz, $\left.\mathrm{CDCl}_{3}\right) \delta 8.11(\mathrm{~d}, J=7.5 \mathrm{~Hz}, 2 \mathrm{H}), 8.01-7.94(\mathrm{~m}, 6 \mathrm{H})$, $7.91(\mathrm{~d}, J=7.5 \mathrm{~Hz}, 2 \mathrm{H}), 7.88(\mathrm{~d}, J=7.5 \mathrm{~Hz}, 2 \mathrm{H}), 7.85(\mathrm{~d}, J=7.0 \mathrm{~Hz}, 2 \mathrm{H}), 7.77(\mathrm{~d}, J=7.0 \mathrm{~Hz}$ 2H), $7.56(\mathrm{~d}, J=7.5 \mathrm{~Hz}, 2 \mathrm{H}), 7.54-7.44(\mathrm{~m}, 4 \mathrm{H}), 7.43-7.22(\mathrm{~m}, 19 \mathrm{H}), 7.17-7.10(\mathrm{~m}, 4 \mathrm{H}), 6.26(\mathrm{t}$, $\left.J_{3,2}=J_{3,4}=10.0 \mathrm{~Hz}, 1 \mathrm{H}, \mathrm{H}-3\right), 6.22\left(\mathrm{~d}, J_{1,2}=3.5 \mathrm{~Hz}, 1 \mathrm{H}, \mathrm{H}-1\right), 6.09\left(\mathrm{t}, J_{4,3}=J_{4,5}=10.0 \mathrm{~Hz}, 1 \mathrm{H}\right.$, $\mathrm{H}-4), 6.01\left(\mathrm{~d}, J_{3^{\prime}, 4^{\prime}}=6.5 \mathrm{~Hz}, 1 \mathrm{H}, \mathrm{H}-3^{\prime}\right), 5.95\left(\mathrm{t}, J_{4^{\prime}, 3^{\prime}}=J_{4^{\prime}, 5^{\prime}}=6.5 \mathrm{~Hz}, 1 \mathrm{H}, \mathrm{H}-4^{\prime}\right), 5.47\left(\mathrm{dd}, J_{2,1}=\right.$ $\left.3.5 \mathrm{~Hz}, J_{2,3}=10.0 \mathrm{~Hz}, 1 \mathrm{H}, \mathrm{H}-2\right), 4.70-4.58\left(\mathrm{~m}, 5 \mathrm{H}, \mathrm{H}-1^{\prime}(2 \mathrm{H}), \mathrm{H}-5^{\prime}, \mathrm{H}-6^{\prime}(2 \mathrm{H})\right), 4.53$ (d, $J_{5,4}=$ $10.0 \mathrm{~Hz}, 1 \mathrm{H}, \mathrm{H}-5), 3.85$ (d, $\left.J_{6 \alpha, 6 \beta}=12.0 \mathrm{~Hz}, 1 \mathrm{H}, \mathrm{H}-6 \alpha\right), 3.76\left(\mathrm{~d}, J_{6 \beta, 6 \alpha}=12.0 \mathrm{~Hz}, 1 \mathrm{H}, \mathrm{H}-6 \beta\right), 1.08$ (s, 9H, H-tBu); ${ }^{13} \mathrm{C}$ NMR $\left(125 \mathrm{MHz}, \mathrm{CDCl}_{3}\right) \delta 165.79,165.73,165.66,165.30,165.27,165.19$, 164.67, 135.67, 135.45, 133.46, 133.35, 133.14, 133.00, 132.96, 132.89, 132.81, 130.03, 129.75, $129.74,129.69,129.60,129.57,129.52,129.39,129.36,129.31,129.18,128.74,128.64,128.55$, 128.36, 128.21, 128.13, 127.56, 127.43, 104.14 (C-2'), 90.77 (C-1), 78.72 (C-5'), 77.15 (C-3'), 75.85 (C-4'), 71.59 (C-5), 71.54 (C-2), 70.66 (C-3), 68.02 (C-4), 64.84 (C-1'), 64.12 (C-6'), 61.22 (C-6), 26.66, 19.11; IR ( $\left.\mathrm{cm}^{-1}\right)$ 3070, 2959, 2857, 1732, 1602, 1585, 1492, 1472, 1451, $1428,1315,1269,1177,1160,1107,1070,1026,823,802,739,708,686 ;$ HRMS m/z calcd for $\left(\mathrm{C}_{77} \mathrm{H}_{68} \mathrm{O}_{18} \mathrm{Si}\right) \mathrm{Na}^{+} 1331.4067$, found 1331.4705; $[\alpha]_{D}^{20}=16.2\left(\mathrm{c} 1.00, \mathrm{CHCl}_{3}\right)$.

\section{6. $2,3,4,1^{\prime}, 3^{\prime}, 4^{\prime}, 6^{\prime}$-hepta- $O$-benzoylsucrose (1)}

To a $1 \mathrm{~L}$ flask charged with $400 \mathrm{~mL} \mathrm{MeOH}$ was added $10.56 \mathrm{~g}(8.06 \mathrm{mmol})$ of $\mathbf{6}$. The mixture was refluxed with vigorous stirring for $10 \mathrm{~min}$. Then to the solution was added $2.08 \mathrm{~mL}$ ( 40.3 mmol, 5 equiv) of $\mathrm{Br}_{2}$ and the mixture was refluxed for another $2 \mathrm{~h}$, after which the reaction was quenched with a saturated sodium hydrosulfite solution. The solvent was removed under reduced pressure. The residue was extracted with $\mathrm{CH}_{2} \mathrm{Cl}_{2}$, dried over $\mathrm{MgSO}_{4}$ and concentrated under reduced pressure. The crude product was purified by flash chromatography $30 \%$ EtOAc in 
hexanes, $R_{f}=0.27$ ), which resulted in $4.78 \mathrm{~g}$ of product ( $4.46 \mathrm{mmol}, 55 \%, 86 \%$ yield based on recovered SM) as a viscous oil (3.79 $\mathrm{g} 6$ was recovered), which foamed upon evacuation (mp 82$\left.88{ }^{\circ} \mathrm{C}\right) .{ }^{1} \mathrm{H}$ NMR $\left(500 \mathrm{MHz}, \mathrm{CDCl}_{3}\right) \delta 8.22(\mathrm{~d}, J=7.5 \mathrm{~Hz}, 2 \mathrm{H}), 8.03-7.96(\mathrm{~m}, 6 \mathrm{H}), 7.86(\mathrm{~d}, J=$ $7.5 \mathrm{~Hz}, 2 \mathrm{H}), 7.84(\mathrm{~d}, J=7.5 \mathrm{~Hz}, 2 \mathrm{H}), 7.80(\mathrm{~d}, J=7.5 \mathrm{~Hz}, 2 \mathrm{H}), 7.61-7.47(\mathrm{~m}, 7 \mathrm{H}), 7.42-7.28(\mathrm{~m}$, $10 \mathrm{H}), 7.25(\mathrm{t}, J=7.5 \mathrm{~Hz}, 2 \mathrm{H}), 7.11(\mathrm{t}, J=7.5 \mathrm{~Hz}, 2 \mathrm{H}), 6.24\left(\mathrm{t}, J_{3,2}=J_{3,4}=10.0 \mathrm{~Hz}, 1 \mathrm{H}, \mathrm{H}-3\right)$, $6.16\left(\mathrm{~d}, J_{1,2}=3.5 \mathrm{~Hz}, 1 \mathrm{H}, \mathrm{H}-1\right), 6.03\left(\mathrm{~d}, J_{3^{\prime}, 4^{\prime}}=6.0 \mathrm{~Hz}, 1 \mathrm{H}, \mathrm{H}-3^{\prime}\right), 6.00\left(\mathrm{t}, J_{4^{\prime}, 3^{\prime}}=J_{4^{\prime}, 5^{\prime}}=6.0 \mathrm{~Hz}\right.$, $\left.1 \mathrm{H}, \mathrm{H}^{\prime} 4^{\prime}\right), 5.53\left(\mathrm{t}, J_{4,3}=J_{4,5}=10.0 \mathrm{~Hz}, 1 \mathrm{H}, \mathrm{H}-4\right), 5.41\left(\mathrm{dd}, J_{2,1}=3.5 \mathrm{~Hz}, J_{2,3}=10.0 \mathrm{~Hz}, 1 \mathrm{H}, \mathrm{H}-\right.$ 2), $4.83\left(\mathrm{dd}, J_{6^{\prime} \alpha, 5^{\prime}}=6.5 \mathrm{~Hz}, J_{6^{\prime} \alpha, 6^{\prime} \beta}=12.0 \mathrm{~Hz}, 1 \mathrm{H}, \mathrm{H}-6^{\prime} \alpha\right), 4.73\left(\mathrm{dd}, J_{6^{\prime} \beta, 5^{\prime}}=5.0 \mathrm{~Hz}, J_{6^{\prime} \beta, 6^{\prime} \alpha}=\right.$ $\left.12.0 \mathrm{~Hz}, 1 \mathrm{H}, \mathrm{H}-6^{\prime} \beta\right), 4.65\left(\mathrm{t}, J_{5^{\prime}, 4^{\prime}}=J_{5^{\prime} 6^{\prime}}=6.0 \mathrm{~Hz}, 1 \mathrm{H}, \mathrm{H}-5^{\prime}\right), 4.62\left(\mathrm{~d}, J_{1^{\prime} \alpha, 1^{\prime} \beta}=12.0 \mathrm{~Hz}, 1 \mathrm{H}, \mathrm{H}-\right.$ $\left.1^{\prime} \alpha\right), 4.56\left(\mathrm{~d}, J_{1^{\prime} \alpha, 1^{\prime} \beta}=12.0 \mathrm{~Hz}, 1 \mathrm{H}, \mathrm{H}-1^{\prime} \beta\right), 4.44\left(\mathrm{~d}, J_{5,4}=10.0 \mathrm{~Hz}, 1 \mathrm{H}, \mathrm{H}-5\right), 3.74\left(\mathrm{dd}, J_{6 \alpha, 6 \beta}=\right.$ $\left.12.0 \mathrm{~Hz}, J_{6 \alpha, 5}=8.0 \mathrm{~Hz}, 1 \mathrm{H}, \mathrm{H}-6 \alpha\right), 3.67-3.61(\mathrm{~m}, 1 \mathrm{H}, \mathrm{H}-6 \beta), 2.82\left(\mathrm{t}, J_{\mathrm{OH}, 6}=7.0 \mathrm{~Hz}, 1 \mathrm{H}, \mathrm{H}-\mathrm{OH}\right)$; ${ }^{13} \mathrm{C}$ NMR $\left(125 \mathrm{MHz}, \mathrm{CDCl}_{3}\right) \delta 166.20,165.95,165.67,165.48,165.44,165.39,165.25,133.59$, $133.51,133.21,133.14,133.07,132.99,130.17,129.95,129.85,129.82,129.72,129.68,129.51$, $129.34,129.18,129.10,128.82,128.69,128.61,128.54,128.45,128.42,128.31,128.29,128.27$, 128.22, 128.18, 104.25 (C-2'), 90.57 (C-1), 78.96 (C-5'), 77.29 (C-4'), 76.20 (C-3’), 71.66 (C5), 71.20 (C-2), 69.83 (C-3), 69.11 (C-4), 64.85 (C-1'), 64.35 (C-6'), $60.92(\mathrm{C}-6)$; IR ( $\left.\mathrm{cm}^{-1}\right)$ $3530,3063,2961,1729,1602,1584,1492,1451,1379,1316,1270,1178,1095,1070,1026$,

854, 803, 708, 686; HRMS m/z calcd for $\left(\mathrm{C}_{61} \mathrm{H}_{50} \mathrm{O}_{18}\right) \mathrm{Na}^{+} 1093.2889$, found 1093.2878; $[\alpha]_{D}^{20}=$ $20.6\left(\right.$ c $\left.1.00, \mathrm{CHCl}_{3}\right)$.

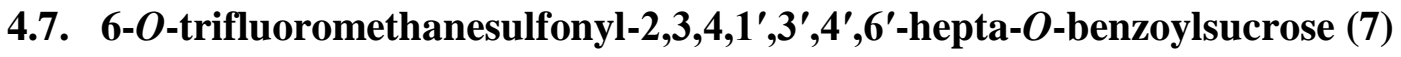

To a solution of $3.93 \mathrm{~g}$ of $\mathbf{1}(3.67 \mathrm{mmol}, 0.05 \mathrm{M})$ in $73 \mathrm{~mL}$ of dichloromethane $(0.05 \mathrm{M})$ at -78 ${ }^{\circ} \mathrm{C}$ was added $765 \mu 1$ of 2,6-lutidine $(6.60 \mathrm{mmol}, 1.8$ equiv) and followed by $925 \mu 1$ trifluoromethanesulfonic anhydride (5.50 mmol, 1.5 equiv). The reaction was stirred for $20 \mathrm{~min}$, 
and then the solution was filtered through a short plug of silica gel and washed with $\mathrm{CH}_{2} \mathrm{Cl}_{2}$. The resulting solution was concentrated under reduced pressure, and then purified by flash chromatography $\left(\mathrm{SiO}_{2}, 30 \%\right.$ EtOAc/hexanes, $\left.\mathrm{R}_{\mathrm{f}}=0.40\right)$, afford $3.8 \mathrm{~g}$ of 7 as a white solid foam (86\% yield); mp 87-92 ${ }^{\circ} \mathrm{C} ;{ }^{1} \mathrm{H}$ NMR $\left(500 \mathrm{MHz}, \mathrm{CDCl}_{3}\right) \delta 8.18(\mathrm{~d}, J=7.5 \mathrm{~Hz}, 2 \mathrm{H}), 8.05-7.96(\mathrm{~m}$, $6 \mathrm{H}), 7.88-7.82(\mathrm{~m}, 4 \mathrm{H}), 7.79(\mathrm{~d}, J=8.0 \mathrm{~Hz}, 2 \mathrm{H}), 7.61(\mathrm{t}, J=7.5 \mathrm{~Hz}, 1 \mathrm{H}), 7.57-7.46(\mathrm{~m}, 6 \mathrm{H})$, 7.42-7.27 (m, 10H), $7.24(\mathrm{t}, J=7.5 \mathrm{~Hz}, 2 \mathrm{H}), 7.11(\mathrm{t}, J=7.5 \mathrm{~Hz}, 2 \mathrm{H}), 6.26-6.20(\mathrm{~m}, 2 \mathrm{H}, \mathrm{H}-1$, H3), 6.00-5.95 (m, 2H, H-3', H-4'), 5.66 (t, $\left.J_{4,3}=J_{4,5}=10.0 \mathrm{~Hz}, 1 \mathrm{H}, \mathrm{H}-4\right), 5.39$ (dd, $J_{2,1}=3.5 \mathrm{~Hz}$, $\left.J_{2,3}=10.0 \mathrm{~Hz}, 1 \mathrm{H}, \mathrm{H}-2\right), 4.79-4.62$ (m, 6H, H-5, H-1', H-5', H-6'), 4.53 (d, $J_{6 \alpha, 6 \beta}=11.0 \mathrm{~Hz}, 1 \mathrm{H}$, $\mathrm{H}-6 \alpha), 4.46\left(\mathrm{~d}, J_{6 \alpha, 6 \beta}=11.0 \mathrm{~Hz}, 1 \mathrm{H}, \mathrm{H}-6 \beta\right) ;{ }^{13} \mathrm{C} \mathrm{NMR}\left(125 \mathrm{MHz}, \mathrm{CDCl}_{3}\right) \delta 165.98,165.50$, $165.41,165.35,165.31,165.17,164.90,133.69,133.61,133.54,133.23,133.14,133.09,129.99$, $129.84,129.81,129.79,129.68,129.60,129.52,129.30,129.05,128.81,128.78,128.70,128.56$, 128.50, 128.44, 128.32, 128.29, 128.20, 128.18, 118.43 (q, $J=317.92 \mathrm{~Hz}, 1 \mathrm{C}), 104.92$ (C-2'), 90.82 (C-1), 79.21 (C-5'), 77.63 (C-3'), 76.25 (C-4'), 72.83 (C-6), 70.86 (C-2), 69.57 (C-3), 68.63 (C-5), 67.94 (C-4), 64.52 (C-1'), 63.83 (C-6’); IR ( $\left.\mathrm{cm}^{-1}\right)$ 3064, 2958, 1729, 1602, 1585, $1492,1452,1419,1316,1267,1214,1178,1144,1094,1070,1026,983,935,845,803,707$, 686; HRMS m/z calcd for $\left(\mathrm{C}_{62} \mathrm{H}_{49} \mathrm{~F}_{3} \mathrm{O}_{20} \mathrm{~S}\right) \mathrm{Na}^{+} 1225.2382$, found $1225.2368 ;[\alpha]_{D}^{20}=19.6$ (c 1.00 , $\left.\mathrm{CHCl}_{3}\right)$.

\subsection{6-deoxy-6-fluoro- $2,3,4,1^{\prime}, 3^{\prime}, 4^{\prime}, 6^{\prime}$-hepta- $O$-benzoylsucrose (2)}

To a solution of $98.3 \mathrm{mg}$ of $7(0.0817 \mathrm{mmol})$ in $8.2 \mathrm{~mL}$ of $\mathrm{CH}_{3} \mathrm{CN}$ at room temperature was added $7.1 \mathrm{mg}$ (0.1226 mmol, $1.5 \mathrm{eq})$ of KF, followed by $46 \mathrm{mg} \mathrm{K222} \mathrm{(0.1226} \mathrm{mmol,} 1.5$ equiv). The solution was then refluxed for $10 \mathrm{~min}$. Once the reaction was complete, the solvent was removed by reduced pressure, and the product was purified by flash chromatography $\left(\mathrm{SiO}_{2}, 30 \%\right.$

EtOAc in hexanes, $\left.\mathrm{R}_{\mathrm{f}}=0.38\right)$, to afford $78 \mathrm{mg}$ of a semisolid $(0.0727 \mathrm{mmol}, 89 \%$ yield $) .{ }^{1} \mathrm{H}$ 
$\operatorname{NMR}\left(500 \mathrm{MHz}, \mathrm{CDCl}_{3}\right) \delta 8.20(\mathrm{~d}, J=7.5 \mathrm{~Hz}, 2 \mathrm{H}), 8.04-7.96(\mathrm{~m}, 6 \mathrm{H}), 7.85(\mathrm{~d}, J=7.5 \mathrm{~Hz}, 2 \mathrm{H})$, $7.83(\mathrm{~d}, J=7.0 \mathrm{~Hz}, 2 \mathrm{H}), 7.76(\mathrm{~d}, J=8.0 \mathrm{~Hz}, 2 \mathrm{H}), 7.60(\mathrm{t}, J=7.5 \mathrm{~Hz}, 1 \mathrm{H}), 7.57-7.47(\mathrm{~m}, 6 \mathrm{H})$, 7.43-7.29 (m, 10H), 7.26 (t, $J=7.5 \mathrm{~Hz}, 2 \mathrm{H}), 7.12$ (t, $J=7.5 \mathrm{~Hz}, 2 \mathrm{H}), 6.24-6.17(\mathrm{~m}, 2 \mathrm{H}, \mathrm{H}-1, \mathrm{H}-$ 3), 6.05-5.96 (m, 2H, H-3', H-4'), $5.64\left(\mathrm{t}, J_{4,3}=J_{4,5}=10.0 \mathrm{~Hz}, 1 \mathrm{H}, \mathrm{H}-4\right), 5.40\left(\mathrm{dd}, J_{2,1}=3.5 \mathrm{~Hz}\right.$, $\left.J_{2,3}=10.0 \mathrm{~Hz}, 1 \mathrm{H}, \mathrm{H}-2\right), 4.76-4.70$ (m, 2H, H-6'), 4.66-4.38 (m, 6H, H-5, H-6, H-1', H-5'); ${ }^{13} \mathrm{C}$ NMR $\left(125 \mathrm{MHz}, \mathrm{CDCl}_{3}\right) \delta 166.05,165.63,165.46,165.42,165.40,165.29,165.04,133.60$, $133.59,133.44,133.25,133.10,133.09,133.03,130.16,129.87,129.84,129.73,129.72,129.58$, $129.43,129.20,129.07,128.88,128.73,128.66,128.55,128.47,128.44,128.33,128.31,128.29$, 128.26, 128.21, 104.20 (C-2'), 90.39 (C-1), 80.89 (d, $J=173.75 \mathrm{~Hz}, 1 \mathrm{C}, \mathrm{C}-6), 78.78$ (C-5'), 77.39 (C-3’), 75.90 (C-4'), 71.07 (C-2), 70.02 (d, $J=18.75$ Hz, 1C, C-5), 70.01 (C-3), 68.08 (d, $\mathrm{J}=6.25 \mathrm{~Hz}, 1 \mathrm{C}, \mathrm{C}-4), 65.03\left(\mathrm{C}-1^{\prime}\right), 64.14\left(\mathrm{C}-6^{\prime}\right) ;{ }^{19} \mathrm{~F}$ NMR $\left(235 \mathrm{MHz}, \mathrm{CDCl}_{3}\right) \delta-232.81(\mathrm{dt}, J=$ 23.5, 47.0 Hz, 1F); IR (cm $\left.{ }^{-1}\right) 3063,2961,1728,1602,1584,1492,1451,1377,1315,1265$, 1177, 1095, 1070, 1026, 802, 707, 686; HRMS m/z calcd for $\left(\mathrm{C}_{61} \mathrm{H}_{49} \mathrm{FO}_{17}\right) \mathrm{Na}^{+} 1095.2846$, found 1095.2836; $[\alpha]_{D}^{20}=22.6\left(\mathrm{c} 1.00, \mathrm{CHCl}_{3}\right)$.

\subsection{6-deoxy-6-fluorosucrose (8)}

To a solution of $1.07 \mathrm{~g}(1.00 \mathrm{mmol})$ of 2 in $20 \mathrm{~mL} \mathrm{MeOH}(0.05 \mathrm{M})$ was added $138 \mathrm{mg} \mathrm{K} \mathrm{CO}_{3}$ (1.00 mmol, 1.0 equiv) at rt. The solution was then refluxed for $5 \mathrm{~min}$. The reaction was monitored with TLC. The solvent was then removed under reduced pressure. The product was purified by flash chromatography $\left(\mathrm{SiO}_{2}, 10 \% \mathrm{H}_{2} \mathrm{O}\right.$ in $\left.\mathrm{CH}_{3} \mathrm{CN}, \mathrm{R}_{\mathrm{f}}=0.28\right)$, to afford $346 \mathrm{mg}$ of $\mathbf{8}$ (1.00 mmol, $100 \%$ yield). The product was recrystallized from water, mp $181-182{ }^{\circ} \mathrm{C} ;{ }^{1} \mathrm{H}$ NMR $\left(500 \mathrm{MHz}, \mathrm{D}_{2} \mathrm{O}\right) \delta 5.43\left(\mathrm{~d}, J_{1,2}=3.5 \mathrm{~Hz}, 1 \mathrm{H}, \mathrm{H}-1\right), 4.78(\mathrm{~s}, 7 \mathrm{H}, \mathrm{OH}), 4.69$ (dd, $J_{6 \alpha, 6 \beta}=11.0 \mathrm{~Hz}$, $\left.J_{6 \alpha, 5}=3.5 \mathrm{~Hz}, 1 \mathrm{H}, \mathrm{H}-6 \alpha\right), 4.63\left(\mathrm{~d}, J_{6 \alpha, 6 \beta}=11.0 \mathrm{~Hz}, 1 \mathrm{H}, \mathrm{H}-6 \beta\right), 4.22\left(\mathrm{~d}, J_{3^{\prime}, 4^{\prime}}=9.0 \mathrm{~Hz}, 1 \mathrm{H}, \mathrm{H}-3^{\prime}\right)$, 4.08-3.97 (m, 2H, H-5, H-4'), 3.92-3.86 (m, 1H, H-5'), 3.84-3.73 (m, 3H, H-3, H-6'), 3.67 (s, 
$\left.2 \mathrm{H}, \mathrm{H}-1^{\prime}\right), 3.58\left(\mathrm{dd}, J_{2,1}=3.5 \mathrm{~Hz}, J_{2,3}=10.0 \mathrm{~Hz}, 1 \mathrm{H}, \mathrm{H}-2\right), 3.50\left(\mathrm{t}, J_{4,3}=J_{4,5}=10.0 \mathrm{~Hz}, 1 \mathrm{H}, \mathrm{H}-\right.$ 4); ${ }^{13} \mathrm{C}$ NMR (125 MHz, $\left.\mathrm{D}_{2} \mathrm{O}\right) \delta 106.43$ (C-2'), 94.81 (C-1), 85.01 (d, $\left.J=167.5 \mathrm{~Hz}, 1 \mathrm{C}, \mathrm{C}-6\right)$, 84.07 (C-5'), 78.95 (C-3'), 76.73 (C-4'), 75.06 (C-3), 74.02 (d, J = 17.5 Hz, 1C, C-5), 73.63 (C2), $71.12(\mathrm{~d}, J=7.5 \mathrm{~Hz}, 1 \mathrm{C}, \mathrm{C}-4), 65.11$ (C-6'), $63.95\left(\mathrm{C}-1^{\prime}\right) ;{ }^{19} \mathrm{~F} \mathrm{NMR}\left(235 \mathrm{MHz}, \mathrm{D}_{2} \mathrm{O}\right) \delta$ $234.83(\mathrm{dt}, J=28.2,47.0 \mathrm{~Hz}, 1 \mathrm{~F})$; IR $\left(\mathrm{cm}^{-1}\right) 3576,3338,3008,2975,2935,1650,1454,1435$, 1411, 1388, 1365, 1346, 1332, 1277, 1235, 1207, 1159, 1126, 1112, 1068, 1049, 1014, 988, 939, 921, 862, 735, 680; HRMS m/z calcd for $\left(\mathrm{C}_{12} \mathrm{H}_{21} \mathrm{FO}_{10}\right) \mathrm{Na}^{+} 367.1011$, found 367.1010 ; $[\alpha]_{D}^{20}=$ $60.0\left(\mathrm{c} 1.00, \mathrm{H}_{2} \mathrm{O}\right),[\alpha]_{D}^{20}=63.4(\mathrm{c} 1.00, \mathrm{MeOH})$

\section{Acknowledgements}

This work was generously supported by a grant from the Department of Energy (DESC0002040). We thank Ms. Carissa S. Hampton (University of Missouri-Columbia) for assistance in preparing this manuscript.

\section{References}

1 Gaddam, V.; Harmata, M. Carbohydr. Res. 2013, 369, 38-41.

2 Ying, W.; Gaddam, V.; Harmata, M. Org. Lett. 2013, 15, 2723-2725.

3 Rotsch, D.; Brossard, T.; Ying, W.; Gaddam, V.; Harmata, M.; Robertson, J. D.; Swyers, X.; Braun, D. M.; Jurisson, S. S., manuscript in preparation.

4 Eklund, S. H.; Robyt, J. F. Carbohydr. Res. 1988, 177, 253-258.

5 (a) Andrade, M. M.; Barros, M. T. Tetrahedron 2004, 60, 9235-9243. (b) Karl, H.; Lee, C. K.; Khan, R. Carbohydr. Res. 1982, 101, 31-38.

6 Batten, R. J.; Dixon, A. J.; Taylor, R. J. K.; Newton, R. F. Synthesis 1980, 234-236.

7 Barros, M. T.; Maycock, C. D.; Thomassigny, C. Synlett 2001, 1146-1148. 

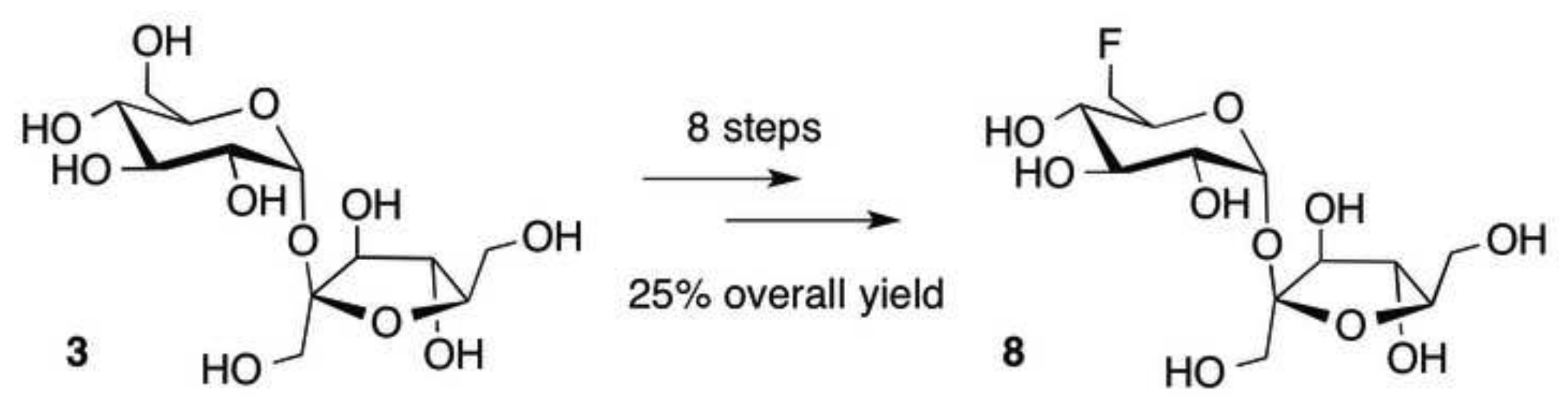

8

.

